Anuario Latinoamericano Ciencias Políticas

y Relaciones Internacionales

vol. 3, 2016

pp. $125-141$

\section{El Concordato colombiano y la jurisprudencia de la Corte Constitucional}

\section{The Colombian Concordat and the jurisprudence of the Constitutional Court}

\author{
Marta Zuzanna Osuchowska ${ }^{1}$ \\ UNIVERSIDAD DE CARDENAL STEFAN WYSZYŃSKI DE VARSOVIA \\ POLONIA \\ $\triangle$ m.osuchowska@uksw.edu.pl
}

\begin{abstract}
RESUMEN
El 12 de julio de 1973 se firmó el Concordato vigente entre la Santa Sede y la República de Colombia. La Constitución de 1991 planteó la cuestión de la conformidad o no conformidad del Concordato con el nuevo texto constitucional. La Corte Constitucional en la sentencia C-027 de 5 de febrero de 1993 declaró inconstitucionales varios artículos del Concordato. En la práctica, el Concordato se sigue aplicando en la mayoría de sus artículos. La razón principal es que en los últimos años, las leyes se han vuelto totalmente compatibles con las disposiciones generales del Concordato, y también es aplicable a todas las religiones, en particular en la Ley Estatutaria de Libertad Religiosa de 1994.
\end{abstract}

PALABRAS CLAVE: concordato, Colombia, constitución, Corte Constitucional, sentencia.

\begin{abstract}
On 12 July 1973 the current Concordat between the Holy See and the Republic of Colombia was signed. The 1991 Constitution raised the question of conformity or nonconformity of the Concordat with the new constitutional text. The Constitutional Court declared unconstitutional a number of articles of the Concordat in Decision C-027, 1993. In practice, the Concordat continues to be applied in most of its articles. The main reason is that in recent years, laws have been rendered wholly compatible

1 Doctora en Derecho. Profesora auxiliar en la Cátedra de Derecho Eclesiástico del Estado y Derecho Concordatario, Facultad de Derecho y Administración, Universidad de Cardenal Stefan Wyszyński de Varsovia (UKSW). Autora y coautora de varios libros y numerosos artículos sobre derecho eclesiástico del Estado, especialmente relacionados con la región de América Latina. Principales líneas de investigación: análisis de la jurisprudencia de los tribunales y órganos nacionales e internacionales en materia de protección del derecho a la libertad religiosa, régimen jurídico de las asociaciones religiosas, personalidad jurídica de las comunidades religiosas.
\end{abstract}


Dossier América Latina: política y religión with the general provisions of the Concordat, and also applicable to all faiths, particularly in the Religious Freedom Act of 1994.

KEYWORDS: concordat, Colombia, constitution, Constitutional Court, sentence.

\section{Introducción}

Colombia tiene una larga tradición de firmar acuerdos con la Santa Sede. Los primeros concordatos se celebraron poco después de la independencia de este país. Por otra parte, en los años por medio de tratados internacionales se arreglaban cuestiones concernientes, sobre todo, a la misión. El concordato de 1973 es una muestra de continuación de las relaciones formalizadas iniciadas en 1887 entre la República de Colombia y la Santa Sede. La reforma en este ámbito fue adoptada a raíz de la Ley 20 de 1974 que implantó en el orden legal colombiano el mencionado Concordato, o sea más de 20 años antes que la Ley Básica vigente en este país de 1991. La modificación de la Constitución de Colombia implantó una serie de nuevas instituciones, como el Tribunal Constitucional responsable también de controlar acuerdos internacionales. Sin embargo, faltan bases para que este órgano estudiara tratados que se habían puesto en vigor antes de la reforma constitucional de 1991. Pese a ello, violando el susodicho principio, en 1993 el Tribunal Constitucional de Colombia emitió un fallo que causó la inviabilidad de más de la mitad de las leyes del Concordato. Hasta hoy en día en la doctrina colombiana, entre los representantes de la ley constitucional e internacional no hay acuerdo con respecto a la viabilidad del Concordato y su Estado (Prieto 2008: 78-85).

En los años siguientes el Concordato contribuyó a la formación y fortalecimiento del modelo colombiano de relaciones entre el Estado y las asociaciones religiosas. Después de 1993, a raíz de poner en vigor muchas de sus normas como principios para la legislación ordinaria, en parte se logró superar las dificultades que aparecieron después de encontrar inaplicabilidad de más de la mitad del tratado. Sin embargo, con el fin de lograr una resolución definitiva de todas las dudas relacionadas con el Concordato colombiano habría que llevar a cabo una revisión a fondo de este acuerdo internacional.

\section{El Concordato colombiano de 1973}

El 12 de julio de 1973 la República de Colombia firmó con la Santa Sede el Concordato que está vigente hasta hoy en día. Fue aprobado (junto con el protocolo final) con la Ley 20 de 1974 que al mismo tiempo suprimió la aplicabilidad del Concordato de 1887 (Martín de Agar 2000: 126-145, Navarro Floria 2011). El Concordato colombiano fue el primer acuerdo firmado por la Santa Sede después del Concilio Vaticano II. Las modificaciones que se introdujeron en 
este campo durante el sínodo causaron la necesidad de revisión de los acuerdos que estaban vigentes en aquel entonces. Para una parte de los representantes de la doctrina las modificaciones de la concepción del Concordato después del Concilio Vaticano II causaron una crisis en el campo del derecho canónico, sin embargo, esto no confirma ni el acuerdo firmado con Colombia, ni una serie de otros acuerdos de esta índole que se siguen celebrando por la Santa Sede (Restrepo Uribe 1973, Vázquez Carrizosa 1973, Holguín 1973, Eguren 1974, Monroy Cabra 1975, Lozano Simonelli 1981, Preciado Agudelo 1989).

En caso de Colombia, el Concordato de 1973, independientemente de su importancia histórica y de la influencia en las relaciones entre la Santa Sede y Colombia en la segunda mitad del siglo XX, dio también comienzo a la discusión sobre el ámbito de la aplicabilidad del derecho internacional en el sistema legal interno. El conflicto que surgió después de poner en vigor la nueva Constitución de 1991 tiene, de todas formas, un alcance más amplio. Abarca también - y quizás sobre todo - la cuestión de admisibilidad del proceso de verificación de los acuerdos vigentes internacionales a la luz de las leyes de una nueva Ley Básica.

El Concordato de 1973 refleja la situación y la época en los que fue firmado, por eso puede ser calificado como un acuerdo transitorio. Pues, en realidad, muchas de sus normas conciernen a los llamados casos mixtos al referirse, al mismo tiempo, a los acuerdos anteriores. Una parte de los artículos están directamente relacionados con los diferentes aspectos de una situación particular de Colombia y constituyen un ejemplo de principios elaborados por el Concilio Vaticano II. ${ }^{2}$ Su influencia se aprecia también en otros aspectos, por ejemplo, en la renuncia a la declaración de la religión católica como confesión del Estado, lo cual era típico de los concordatos de antes del Concilio. En vez de esta fórmula, en el art. I se especificó que el Estado, respetando los sentimientos tradicionales de los católicos de la nación colombiana, reconoce la religión católica, apostólica y romana como un elemento básico del bien común e integral para el desarrollo de la comunidad nacional. Además, este mismo artículo de forma directa reconoce el derecho a la libertad religiosa de los católicos y de los fieles de otras confesiones, al considerar que el Estado garantiza un pleno reconocimiento de los derechos religiosos. ${ }^{3}$

A la luz de las normas del derecho internacional, la validez del Concordato colombiano, como documento elaborado con corrección de acuerdo con las leyes internas, implantado en el sistema de fuentes del derecho nacional no

2 Cfr. N. 76 Constitución Pastoral "Gaudium et spes" sobre la Iglesia en el mundo actual, p. ej. la cooperación con el Estado en los servicios de educación y promoción social (art. V); cargar de los programas de educación en zonas marginadas (art. XIII); promoción de las condiciones humanas y sociales de la población indígena y de los residentes en zonas marginadas (art. VI); cooperación en la defensa y promoción del patrimonio artístico y cultural (art. XXVIII).

3 „El Estado garantiza a la Iglesia Católica y a quienes a ella pertenecen el pleno goce de sus derechos religiosos, sin perjuicio de justa libertad religiosa de las demás confesiones y de sus miembros lo mismo que de todo ciudadano".
El Concordato colombiano y la jurisprudencia de la Corte Constitucional

Marta Zuzanna Osuchowska 
Dossier América Latina: política y religión despierta ninguna duda. ${ }^{4}$ Sin embargo, estas aparecieron después de poner en vigor la Constitución de 1991, ya que se planteó la cuestión de compatibilidad del Concordato con la nueva Ley Básica (Martín de Agar, Uribe Blanco 1998: 635-643, Prieto 1998, Prieto 2001: 83-93).

De acuerdo con la Convención de Viena, una parte no podrá recurrir a las disposiciones de su derecho interno como justificación del incumplimiento del tratado y al hecho de que su consentimiento en obligarse por el tratado no es válido, ya que se ha expresado en violación de las disposiciones de derecho interno concernientes a la competencia para celebrar tratados, a menos que esta violación fuera evidente (objetivamente evidente para cualquier Estado que procede en la materia conforme a la práctica usual y de buena fe), y esto estaba relacionado a las normas relativas a su derecho interno de importancia fundamental. ${ }^{5}$ Estas disposiciones se complementaron en el derecho colombiano con la ley de la norma constitucional indispensable en la formación de las relaciones exteriores del Estado, entre otros, el reconocimiento de los principios del derecho internacional. ${ }^{6}$ Tampoco podemos hacer caso omiso de la jurisprudencia de la Corte Constitucional, cuyas tareas incluyen, entre otros, probar la constitucionalidad de los acuerdos internacionales antes de su ratificación ${ }^{7}$, pero este control no puede cubrir los tratados ya ratificados, como en el caso del Concordato de $1973 .^{8}$

Independientemente de lo anterior, la Corte Constitucional en la sentencia C-027 de 5 de febrero de 1993 declaró inconstitucional casi la mitad de los artículos del Concordato. ${ }^{9}$ En esta resolución también se rechazó el argumento

${ }^{4}$ De conformidad con el plazo de diez años, el 2 de julio de 1985 tuvo lugar el canje de notas, en Bogotá, entre el Nuncio Apostólico y el Ministro de Relaciones Exteriores. Teniendo en cuenta la cláusula, por iniciativa del Gobierno colombiano, a la Convención de Viena sobre Derecho de los Tratados, que había sido ratificada por Colombia y la Santa Sede: "Queda evidentemente entendido que el Concordato, como Tratado Internacional, continúa regido para todos sus efectos por las normas del Derecho Internacional General y por la Convención de Viena sobre el Derecho de los Tratados".

5 Art. 27 y 46 Convención de Viena sobre Derecho de los Tratados.

6 Art. 9 Constitución de 1991: „Las relaciones exteriores del Estado se fundamentan en la soberanía nacional, en el respeto a la autodeterminación de los pueblos y en el reconocimiento de los principios del derecho internacional aceptados por Colombia".

7 Art. 241 n. 10 Constitución de 1991: „A la Corte Constitucional se le confía la guarda de la integridad y supremacía de la Constitución, en los estrictos y precisos términos de este artículo. Con tal fin, cumplirá las siguientes funciones [...] 10. Decidir definitivamente sobre la exequibilidad de los tratados internacionales y de las leyes que los aprueben. Con tal fin, el Gobierno los remitirá a la Corte, dentro de los seis días siguientes a la sanción de la ley. Cualquier ciudadano podrá intervenir para defender o impugnar su constitucionalidad. Si la Corte los declara constitucionales, el Gobierno podrá efectuar el canje de notas; en caso contrario no serán ratificados. Cuando una o varias normas de un tratado multilateral sean declaradas inexequibles por la Corte Constitucional, el Presidente de la República sólo podrá manifestar el consentimiento formulando la correspondiente reserva".

8 Sentencia T-998 de 2002.

9 Concretamente fueron declarados inconstitucionales los artículos VI (colaboración en los planes de desarrollo en zonas marginadas); IX (atribución al Estado de las causas de separación 
de la falta de una base jurídica para el confirmar la compatibilidad de la nueva Constitución con los tratados ya ratificados antes de su entrada en vigor en virtud de la Ley Básica anterior.

Los principios establecidos en la parte dispositiva C-027 cambiaron rápidamente la línea existente de la jurisprudencia; volvió también el argumento relativo a la incompatibilidad del acuerdo con las normas constitucionales para el examen del derecho internacional por parte del Tribunal. Ahora es ampliamente aplicable la opinión de que esta disposición sólo se refiere a las consecuencias para el futuro y no se aplica a los tratados aprobados y puestos en vigor antes de la Constitución de 1991 como en el caso del Concordato. ${ }^{10}$

El debate sobre el alcance de la actividad de la parte dispositiva C-027 sigue abierto. Por una parte, no hay duda con respecto a la plena validez del Concordato en el orden jurídico colombiano ${ }^{11}$, sin embargo, en relación a los artículos que fueron declarados inconstitucionales hay que aplicar el principio de cosa juzgada, en el caso de una modificación de la jurisprudencia adoptada en 1993.

Por otro lado, en la jurisprudencia de 1993 podemos encontrar disposiciones que apoyan la inconstitucionalidad del Concordato. Por ejemplo, el fallo C-276 de 1993, además de negar en general la competencia de la Corte Constitucional para estudiar la constitucionalidad y ratificar tratados internacionales, establece que las resoluciones emitidas al respecto eran ineficaces. En el fallo C-225 de 1994 se confirmó la validez del Concordato en el orden jurídico internacional, mientras que se resaltó de forma clara que en el orden jurídico nacional los artículos declarados inconstitucionales no se podían aplicar. Existen también excepciones que confirman la regla que conceden una plena aplicabilidad del Concordato.

La mayoría de las doctrinas están de parte de que el fallo C-027 de 1993 no puede afectar en la validez del Concordato que fue ya ratificado y entró

de cónyuges); XI (contribución económica del Estado a las escuelas católicas); XII (clases de religión en la escuela pública); XIII (contratos para la colaboración en los programas educativos en zonas marginadas); XVI (compromiso de la Santa Sede de transformar las jurisdicciones misionales en diócesis); XVII (asistencia pastoral de las Fuerzas Armadas); XX (normas especiales para los procesos penales de clérigos y religiosos); XXII (asimilación del ejercicio ilegítimo de funciones eclesiásticas a la usurpación de funciones públicas). Fueron declarados inexequibles algunos incisos de otros artículos: disolución del vínculo y matrimonio rato y no consumado (art. VIII); comunicación previa al Gobierno de los nombramientos episcopales (art. XIV); información al Gobierno sobre modificación de circunscripciones eclesiásticas (art. XV); atribución a la Santa Sede de los procesos penales contra Obispos y normas especiales de procedimiento penal para clérigos y religiosos (arts. XIX y XX); contribución económica del Estado para la transformación de jurisdicciones misionales en diócesis (art. XXVI).

${ }^{10}$ Sentencia C-276 de 1993, Sentencia C-567 de 1993.

${ }^{11}$ Sentencia C-088 de 1994: „En lo que corresponde al artículo 11, relativo a la personería jurídica de derecho público eclesiástico de la Iglesia Católica, se trata simplemente de la manifestación del respeto a un derecho adquirido, reconocido por el Concordato suscrito entre el Estado Colombiano y la Santa Sede, y por la jurisprudencia de esta Corte, obviamente amparado por la cosa juzgada constitucional, como quiera que fue juzgado en su oportunidad por esta Corporación".
El Concordato colombiano y la jurisprudencia de la Corte Constitucional

Marta Zuzanna Osuchowska 
Dossier América Latina: política y religión en vigor. Lo apoya el mismo procedimiento de terminación de un acuerdo internacional cuya iniciativa la toma únicamente el poder ejecutivo (Prieto 1996: 807).

En la práctica, el Concordato sigue siendo aplicado en su totalidad. Las causas de ello no hay que buscarlas, de todas formas, en la solución de controversias relacionadas con el fallo C-027, sino en las actividades legislativas posteriores. Las nuevas normas jurídicas para diferentes grupos religiosos eran compatibles con las soluciones previstas para la Iglesia católica en el Concordato, también en los artículos impugnados por la Corte Constitucional. En particular, se trata del derecho legal de 1994 sobre la libertad religiosa.

Cabe señalar que entre la puesta en vigor de la nueva Constitución y todavía antes del fallo C-027 de la Corte Constitucional, ante una nueva situación legal el gobierno propuso una revisión del Concordato que fue celebrada por las dos partes el 20 de noviembre de 1992. El acuerdo es comúnmente conocido como Concordato Sanín Romeo. Sin embargo, el documento no consiguió la aprobación del parlamento. Otros acontecimientos, sobre todo el fallo C-027, suspendieron un proceso normal de aprobación y ratificación del acuerdo. La reforma de 1992 nunca entró en vigor. El procedimiento de su aprobación por el Congreso y la Corte Constitucional se suspendió el 5 de febrero de 1993 a resultas del fallo C-02 de la Corte que vio inconstitucionales la mayoría de las resoluciones del Concordato de 1973 (Ramos Castañeda 2014: 260-284).

\section{La jurisprudencia de la Corte Constitucional colombiana}

Con el fin de llevar a cabo una evaluación subjetiva del fallo C-027 de la Corte Constitucional de 1993 y de las siguientes sentencias, hay que aclarar la situación causada a raíz de la adaptación de la nueva Constitución de 1991 en el ámbito del control constitucional.

La constitución de la República de Colombia de 1991 por primera vez estableció la Corte Constitucional para este país. Se considera que el control de los acuerdos internacionales y de las leyes que los aprueban es preventiva, o sea, tiene lugar antes del intercambio de notas de ratificación. Entonces, el control puede tener lugar sólo en el momento en que la Corte considere tanto el tratado como la ley conformes a la Constitución, lo cual, por su parte, autoriza al gobierno a adoptar otras medidas con el fin de ratificar derechos internacionales. Esta sentencia se emite por la Corte Constitucional como órgano de control legislativo y adquiere fuerza legal de la cosa tratada en el proceso constitucional. ${ }^{12}$

Uno de los primeros casos examinados por la Corte Constitucional fue la compatibilidad de la nueva Ley Básica con el Concordato celebrado en 1973 y vigente desde 1975 en Colombia.

\footnotetext{
${ }^{12}$ Art. 243 de la Constitución de 1991.
} 
La ley que aprueba el Concordato, cuya parte integrante es su texto, fue cuestionada como inconstitucional por varias razones y en relación a muchos de sus puntos, sobre todo, a los que confieren la jurisdicción exclusiva sobre las causas de anulación y disolución del matrimonio canónico. Sin embargo, la Constitución extendió las causas del divorcio y la inexistencia del matrimonio por el aspecto civil en todos los matrimonios, no sólo los canónicos.

Durante la vigencia de la anterior Constitución de 1886 muchas veces se cuestionaron las soluciones del Concordato, tanto en esta materia como en otras cuestiones, al igual que las de otros tratados internacionales ratificados por Colombia, sin embargo, la Corte Suprema que entonces era responsable del control constitucional no examinaba la esencia del caso considerándose siempre incompetente para opinar sobre la constitucionalidad de tratados ratificados debidamente. Justificaba que esto era de responsabilidad del poder ejecutivo en el campo de las relaciones internacionales y con el principio pacta sunt servanda (Prieto 1998: 81-124, Monroy Cabra 2001: 40-43).

En la primera etapa, la Corte declaró sin vacilar que el control establecido en el art. 241 de la Constitución era anterior, automática y definitiva. ${ }^{13}$ Esto quiere decir que este tiene que llevarse a cabo antes del intercambio de las notas de ratificación del tratado internacional. En caso de los acuerdos ya ratificados, la Corte no es competente para examinar su constitucionalidad. Esto corresponde al criterio adoptado por la Corte Suprema que estuvo vigente más de ochenta años.

Por esta razón, desde el mismo principio del establecimiento de la Corte Constitucional, hay un debate sobre las facultades y limitaciones de este órgano para resolver sobre la constitucionalidad del Concordato. El art. 241 de la Constitución que concede a la Corte el derecho a examinar la compatibilidad con el derecho de los tratados internacionales no dice nada directamente sobre los actos ya ratificados de este tipo. Entonces, la posibilidad de debate teoréticamente sigue abierta (Vázquez Carrizosa 1993: 145). A pesar de ello, la aplicación del principio de irretroactividad de la ley en caso de la revisión de la Constitución no es fácil. Esto se aprecia en el ejemplo de los cambios que se producen en la jurisprudencia que concierne a otros principios y situaciones legales, o sea, a la supremacía de la Constitución sobre otras leyes, a las relaciones entre la ley internacional y nacional, etc. En general, todas las dudas al respecto de las cuestiones se pueden resumir en una pregunta: ¿La Corte Constitucional puede determinar la incompatibilidad de la Constitución y de las leyes que aprueban los acuerdos internacionales ratificados antes de que esta Constitución entrara en vigor?

Sin embargo, la mayor parte de la doctrina opinaba que había que continuar la práctica de muchos años de moderarse en resolver tales cuestiones. La Corte Constitucional, teniendo en cuenta su conveniencia en casos concer-

${ }^{13}$ Cfr. Sentencia C-574 de 1992, Sentencia C-477de 1992, Sentencia C-562 de 1992, Sentencia C-589 de 1992.
El Concordato colombiano y la jurisprudencia de la Corte Constitucional

Marta Zuzanna Osuchowska 
Dossier América Latina: política y religión nientes al cuidado de algunas leyes básicas que violan el principio ius cogens, emitió una sentencia extensa en la que se consideró competente para examinar las leyes que aprobaban el Concordato con la Constitución. En este caso, examinó artículo tras artículo, considerando algunos de ellos totalmente compatibles y una parte parcialmente o totalmente inviables, o sea, inconstitucionales. En este caso, estamos ante la llamada inconstitucionalidad sobrevenida. Sin embargo, esto no es definitivo en cuanto a la conveniencia de la sentencia, lo cual muchas veces repitió la Corte Suprema.

Teniendo en cuenta las cuestiones susodichas concernientes, sobre todo, a una larga tradición de la jurisprudencia, resultan comprensibles las cuestiones sobre la sentencia C-027 de 1993. En realidad, por primera vez en la tradición legal colombiana, el órgano responsable de la revisión de la Constitución reconoció su competencia en caso de examinar la constitucionalidad de tratado ratificado internacional con respecto al que nunca hubo ninguna duda en cuanto a los defectos formales de su vigencia.

Los argumentos presentados en la presente sentencia son variados y muchas veces confusos. En general, conciernen a dos cuestiones: supremacía de la Constitución sobre los acuerdos internacionales ${ }^{14}$ y violación por parte de las normas del Concordato de los derechos humanos básicos. ${ }^{15}$

Los artículos del Concordato que fueron considerados incompatibles con la Constitución contenían normas que podían poner a la Iglesia católica en una posición privilegiada con respecto a los demás grupos religiosos. Estas concernían, entre otros, a la colaboración en áreas marginales, al hecho de pronunciarse sobre la separación de los cónyuges, a la ayuda financiera por parte del Estado a las escuelas católicas, a las clases de religión en escuelas públicas, a la enseñanza por contratos en áreas marginales, al ministerio pastoral militar y a la jurisdicción penal para el clero. Algunos fragmentos de la decisión justifican de forma directa la aseveración de arriba.

La conferencia episcopal de Colombia, sin esperar una reacción crítica de la sentencia mencionada, consideró que esta violaba los principios del derecho internacional, en los que está basado el Concordato, o sea, pacta sunt servan-

${ }^{14}$ De acuerdo con el art. 4 de la Constitución de 1991: „La Constitución es norma de normas. En todo caso de incompatibilidad entre la Constitución y la Ley u otra norma jurídica, se aplicarán las disposiciones constitucionales (...)”. La Sentencia C-027 de 1993 afirma: „nuestra Constitución no reconoce la supremacía de los Tratados internacionales sobre la Constitución Política. Por tanto la Carta no autoriza a su guardiana a abstenerse de pronunciar la inexequibilidad de un Tratado que aún perfeccionado viola los postulados fundamentales que estructuran la organización jurídico-política e ideológica del Estado colombiano”.

15 „Si bien es cierto que la Ley 20 de 1974 y su Tratado y Protocolo Final estaban perfeccionados al entrar a regir la nueva Constitución, ellos ofrecen la especial connotación de referirse al jus cogens de derecho internacional que ampara los derechos humanos y los coloca en la cima de la jerarquía normativa internacional. Por esta razón y teniendo en cuenta la integración que debe existir entre el ordenamiento interno de las naciones y el exterior de los Estados (art. 93 C.N.), los actos acusados han de ser examinados a la luz de los tratados internacionales sobre derechos humanos con el fin de verificar si se ajustan o no a ellos". 
da. El convenio de Viena sobre el derecho de los tratados, mostró imposibilidad de recurrir al derecho interno con el fin de incumplir los pactos, etc.; además, señaló un orden legal interno, conforme al cual la Corte se excedió de su competencia jurisdiccional, ya que no tenía facultades para examinar la compatibilidad del derecho de los tratados ya ratificados. Su carácter la Corte lo definió sólo mediante el texto del Concordato, violando en este caso los principios del derecho, pues no tomó en cuenta los aspectos de procedimiento. Los obispos definieron la sentencia de la Corte como una herramienta que violaba los derechos humanos, sobre todo de la libertad religiosa, y de este modo reiteraron que el "Concordato está vigente en toda su extensión". ${ }^{16}$

Poco tiempo después de examinar la compatibilidad del Concordato con la Constitución se planteó la cuestión de la constitucionalidad de la adhesión de Colombia al Tratado de Montevideo. ${ }^{17}$ La Corte se consideró en este caso indebido, dada la ratificación de un acuerdo internacional antes de la entrada en vigor de la Constitución. De este modo se volvió a la práctica de la Corte Suprema que antes de 1991 resolvió en casos de este tipo.

Las sentencias de la Corte Constitucional cobran fuerza legal de cosa juzgada en procesos constitucionales, así que afectan a los criterios adoptados por los tribunales inferiores, pero no modifican la esencia de la cosa ya examinada por la misma Corte, como en el caso del Concordato. La jurisprudencia de la Corte Constitucional adoptada en la sentencia C-027 de 1993 no dura mucho. En sentencias posteriores la Corte cambió su actitud al declarar que no era adecuada para el examen de acuerdos internacionales ratificados. ${ }^{18}$ Pese

${ }^{16}$ Conferencia Episcopal de Colombia, Declaración sobre la Sentencia C-027 de la Corte Constitucional en relación con el Concordato vigente entre la Santa Sede y la República de Colombia: n. 2,Afirmamos que el Concordato de 1973 está en vigor en todas sus partes, puesto que su nulidad, terminación, denuncia o suspensión no ha sido alegada por una Alta Parte contratante en aplicación de la Convención de Viena sobre el Derecho de los Tratados. Esta Convención rige el Concordato en todos sus efectos. Por tanto, el mismo obliga a las partes - Estado colombiano y Santa Sede - y debe continuar siendo cumplido por ellas de buena fe. Con la sentencia de la Corte Constitucional, el Estado colombiano ha desconocido el principio "pacta sunt servanda" (todo pacto debe mantenerse), ha transgredido la prohibición de alegar el derecho interno para incumplir un Tratado y, a su vez, ha incumplido su obligación de no frustrar, antes de su entrada en vigor, el objeto y el fin del Acuerdo suscrito entre la Santa Sede y la República de Colombia en noviembre de 1992"; n. 8:"La Corte Constitucional, consciente de que estaba en tela de juicio su competencia, para asegurarla, en oposición a los principios del derecho, juzgó 1 primero el contenido del Tratado. Es este asunto de máxima gravedad, dado que la Corte, con el pretexto de proteger los derechos humanos, excedió la competencia que para la revisión de los tratados internacionales, en "estrictos y precisos términos", le fija la Constitución. Queda en pie, por tanto, la incompetencia de la Corte para juzgar la constitucionalidad del Concordato y de la ley aprobatoria del mismo".

${ }_{17}$ El Tratado de Derecho Civil Internacional firmado el 12 de febrero de 1889. Colombia lo ratificó oficialmente el 2 de diciembre de 1933.

${ }^{18}$ Las primeras decisiones posteriores a la Sentencia C-027 que asumieron el criterio de la incompetencia están contenidas en los Autos n. 002 del 8 de marzo y 003 del 21 de abril de 1993, relativos a la demanda de inconstitucionalidad contra el Acta de Barahona suscrita en Cartagena el 5 de diciembre de 1991 con Bolivia, Ecuador y Venezuela.
El Concordato colombiano y la jurisprudencia de la Corte Constitucional

Marta Zuzanna Osuchowska 
Dossier América Latina: política y religión al cambio de la jurisprudencia, sigue indiscutible el hecho de la importancia de la cosa juzgada en procedimientos constitucionales. Esto significó que los artículos del Concordato considerados inconstitucionales en 1993 seguían así y no era posible que se expidiera otra sentencia en el mismo caso.

Otra cuestión, también relacionada directamente con el alcance de la resolución de 1993, fue reconocer la conveniencia del poder ejecutivo para iniciar el proceso de renuncia a los compromisos resultantes de los acuerdos internacionales, dejando vigente sólo el mismo tratado (Maya Barroso 2008: 66-67).

De acuerdo con la argumentación del Tribunal, podemos hablar de dos formas de control de la Constitución sobre las leyes que aprueban los tratados internacionales que se complementan recíprocamente. Por una parte, existe una revisión automática de los acuerdos internacionales antes de su ratificación, mientras que, por otra, la revisión tiene aplicación en relación a todas las leyes, así como también a las que se refieren a los tratados ya aprobados, a causa tanto del texto como del procedimiento. ${ }^{19}$

$\mathrm{El}$ argumento definitivo se refiere a la necesidad de garantizar el principio básico, integridad y supremacía de las leyes básicas sobre cualquier otra norma. La interpretación que incorpora el artículo 241 de la Constitución es su consecuencia y define la competencia de la Corte sobre cualquier ley, incluso si se trata de tratados internacionales ya ratificados.

Las consecuencias de tal actitud, definida por la sentencia susodicha, se pueden resumir en los siguientes puntos:

1. En el ámbito del derecho interno, la supremacía de la Constitución quiere decir que los tratados incompatibles con ella no deben ser aplicados por la autoridad, teniendo en cuenta el art. 4 de la Constitución.

2. En relación a que Colombia respeta el principio pacta sunt servanda, ante estos acuerdos internacionales que se reconocieron inconstitucionales, la autoridad se ve obligada a modificar el acuerdo internacional de manera que sea compatible con la Constitución o reformar la ley básica en la materia de compromisos internacionales (González 2002).

3. De acuerdo con el principio de la obligación de cumplir los compromisos que está contenida en el art. 9 de la Constitución, el deber legal de los jueces es aplicar normas internas diferentes a la ley básica de una forma que sea la más adecuada para los compromisos internacionales asumidos por el Estado.

4. La sentencia de inconstitucionalidad de la ley que aprueba el tratado no rompe el compromiso en el ámbito internacional, entonces a la luz del de-

${ }^{19}$ Sentencia C-400 de 1998: „Así, del hecho de que el ordinal 10 expresamente consagre una revisión constitucional previa para todos los nuevos Tratados que Colombia pretenda ratificar, una vez en vigor la Constitución de 1991, en manera alguna podemos inferir que la Carta ha excluido el control sobre los Tratados preconstituyentes por vía de demanda ciudadana, cuando todo Tratado, para poder ser incorporado al ordenamiento colombiano, requiere ser aprobado mediante Ley, y a la Corte se le atribuyó, en el ordinal $4^{\circ}$ del mismo artículo 241, la facultad de decidir sobre las demandas de inconstitucionalidad que presenten los ciudadanos contra todas las leyes, tanto por su contenido material como por vicios de procedimiento en su formación”. 
recho internacional tal sentencia no tiene ningún valor legal, sin más, sigue como problema real la cuestión si el Estado cumple sus compromisos al respecto o no. Como resultado, para la jurisprudencia internacional y la doctrina, la sentencia que cancela un tratado o una ley que lo implanta es solamente un hecho que tiene efectos legales, puede amenazar la responsabilidad internacional del Estado, pero sin influencia alguna en la eficacia internacional, tal tratado no existe.

De acuerdo con la jurisprudencia colombiana de los últimos años se puede confirmar lo que sigue:

1. Según el principio de cosa juzgada en un procedimiento constitucional, la sentencia de la Corte C-027 de 1993 es irrefutable. Esta se puede modificar sólo en caso de una interpretación diferente del art. 241 de la Constitución, y no en relación a la inconstitucionalidad de algunas normas del Concordato vigente.

2. Las normas del Concordato que se consideran inconstitucionales no son factibles en Colombia de acuerdo con el principio de la supremacía de la ley básica. A pesar de ello, siguen siendo una parte del tratado vigente en el orden internacional. Surge un conflicto resultante del hecho de que el acuerdo internacional no se aplica en el país a causa de la incompatibilidad entre la Constitución y la ley que la aprueba. Entonces, ¿Cómo es que este tratado puede estar vigente sin ser aplicable? (Carrizosa 1993: 147)

3. El deber del poder ejecutivo es realizar reformas del Concordato de acuerdo con la Constitución, y de esta forma restablecer la armonía ente el sistema interno y el orden internacional (Prieto 2010: 30-31).

Además, el gobierno de Colombia afirma que el Concordato sigue en vigor hasta en el orden nacional, a pesar de la falta de su revisión o modificación a la luz del derecho internacional. Así que, la sentencia de 1993 no ordenó una inaplicabilidad inmediata y directa en el derecho interno de los artículos considerados incompatibles con la Constitución, sino que más bien afirmó su conformidad con la misma, declarando de este modo que el poder ejecutivo debía implantar actividades que tuvieran por objetivo una reforma del Concordato (de Agar, Blanco 1998: 640).

\section{Las perspectivas de la doctrina}

Una parte de la doctrina considera que el Concordato rige en el orden internacional, pero las normas consideradas inconstitucionales no son una parte del derecho interno. Entonces, no se puede recurrir a ellas ante la administración pública ni ante el tribunal. Una opinión similar la expresó también la Corte en algunas de sus sentencias. En cambio, otros están de parte de la opinión de que la sentencia de la Corte Constitucional sobre el tema del Concordato no es conforme con el derecho (actuando con la violación de las reglas sobre la con-
El Concordato colombiano y la jurisprudencia de la Corte Constitucional

Marta Zuzanna Osuchowska 
Dossier América Latina: política y religión veniencia y violando los derechos internacionales), por eso este tratado está del todo vigente, tanto en el orden internacional como en el interno. Ninguno de los mecanismos previstos para la cancelación o modificación del acuerdo se utilizó en este caso por ninguna de las partes interesadas.

Estas actitudes no parecen cuadrar la una con la otra y su prevalencia en la doctrina indica que en el orden colombiano hay un problema relacionado con la falta de cohesión hasta a nivel constitucional. Servirse de la supremacía de la ley básica que supuestamente violara el Concordato también conformaría la incompetencia con la que la Corte pudiese requerir que se respetaran los principios constitucionales que existen en relaciones internacionales, incluido el principio pacta sunt servanda de los tratados ratificados por Colombia.

Por eso hay que buscar una tercera opinión que intente conciliar las actitudes susodichas y dar una solución a la situación producida. Hay que considerar qué tipo de competencia tiene y ejerce la Corte Constitucional (al emitir leyes aprobatorias) en relación a los acuerdos internacionales ratificados antes que entrara en vigor la Constitución vigente.

Por una parte, es verdad que la modificación de la Constitución no puede significar una retirada unilateral del Estado de los compromisos incluidos en el tratado o modificaciones en su conducta, conforme al art. 27 del Convenio de Viena sobre el derecho de los tratados, lo cual, al menos implícitamente es aceptado por la Corte de Colombia en sentencias posteriores, como, por ejemplo, en caso del Tratado de Montevideo. Por otra parte, no se puede negar que la Corte Constitucional, a la que la ley básica encomienda la protección e integridad del orden constitucional, tiene un título suficiente para estudiar leyes debidas de procedimiento, cualquier norma que sea una parte del orden legal colombiano y que afirmen su conformidad o no con la Constitución, sobre todo, en el campo de los derechos básicos garantizados por la misma. En otro caso se producirá un riesgo de multiplicar discrepancias en el nivel más alto del orden legal colombiano (Prieto 2010: 20-22).

Entonces, el problema principal se basa en el alcance de la obligatoriedad de las sentencias constitucionales en relación a los acuerdos internacionales ya ratificados por el Estado. No se trata de definir directamente la inaplicabilidad de estas normas del tratado que entran en conflicto con la Constitución, sino de afirmar la incompatibilidad con ella del mismo acto internacional. Tal declaración constituiría para el poder ejecutivo la obligación de implantar mecanismos que tengan por objeto reformar tratados en el ámbito presentado para que la Corte los resuelva.

En fin, la Corte al ponerse de parte de la incompatibilidad de algunas cláusulas del tratado con la Constitución, impuso al gobierno la obligación de negociar contrato con la otra parte con el fin de adaptarlo a su orden constitucional.

Esta opinión la comparte la misma sentencia C-027 que declara la inaplicabilidad de uno de los artículos del Concordato sin que se prive el mismo tratado del poder vigente. Esta sentencia de inaplicabilidad hay que interpretarla de acuerdo con todo el sistema jurídico colombiano. 
La misma interpretación fue repetida en las sentencias posteriores y posiciones de varios órganos administrativos con ocasión de resolver los problemas encontrados en relación con la realización y puesta en vigor de esta sentencia de la Corte Constitucional. Por ejemplo, en la opinión del Fiscal General, se encontró la afirmación de acuerdo con la cual en relación con las sentencias de la Corte concernientes a la inconstitucionalidad de los tratados internacionales ratificados por Colombia antes de 1991 hay que, de acuerdo con el derecho internacional, suponer que el convenio o el acuerdo del que se habla sigue en vigor pese a la sentencia, sin embargo, al mismo tiempo se debería indicar a un órgano competente, dado el respeto del derecho interno y de la Constitución, un procedimiento de renegociación de un acuerdo internacional. ${ }^{20}$ También la Secretaría Jurídica del Presidente de la República vio con buenos ojos la afirmación de que la sentencia que declaraba la inaplicabilidad de la ley aprobatoria del tratado no lo priva de la eficacia. Los efectos de tal opinión son, entonces, obligaciones de tomar medidas que lleven a la consolidación de las relaciones entre los dos sujetos de manera que sean conformes con la Ley Básica modificada. ${ }^{21}$

\section{El impacto del Concordato sobre la evolución del derecho eclesiástico del Estado colombiano}

Independientemente de diferentes interpretaciones que pueden surgir en relación con la sentencia C-027 de 1993, está claro que la Constitución de 1991 introdujo en el orden legal colombiano modificaciones relevantes concernientes a los principios que regulan las relaciones entre el Estado y las iglesias. No se trata aquí sólo de la Iglesia católica.

El Concordato que en el sistema anterior podía ser tratado como un instrumento que en cierto sentido aislaba la Iglesia católica, dado su único carácter y consolidación en el sistema legal internacional, en este sentido sirvió de punto de referencia con el fin de ampliar posibilidades de celebrar acuerdos con otros credos. Fue incorporado formalmente al nuevo sistema de derecho común, como un acto de unión a todos. Por tanto, no se puede criticar el tratado como un privilegio legislativo y, al mismo tiempo, proporcionar la oportunidad de un desarrollo de normas bajo los principios generales de derecho.

${ }^{20}$ Concepto del Procurador General de la Nación n. 208 del 7 de junio 1993.

${ }^{21}$ Nota del Ministerio de Relaciones Exteriores M 1339 del 2 de mayo 1994; Concepto de la Secretaría jurídica de la Presidencia de la República, n. 7779 del 24 de noviembre 1993. La Conferencia Episcopal de Colombia se pronunció “Declaración” del 19 de febrero de 1993: „Afirmamos que el Concordato de 1973 está en vigor en todas sus partes, puesto que su nulidad, terminación, denuncia o suspensión no ha sido alegada por una Alta Parte contratante en aplicación de la Convención de Viena sobre el Derecho de los Tratados. Esta Convención rige el Concordato en todos sus efectos. Por tanto, el mismo obliga a las partes - Estado colombiano y Santa Sede y debe continuar siendo cumplido por ellas de buena fe".
El Concordato colombiano y la jurisprudencia de la Corte Constitucional

Marta Zuzanna Osuchowska 
Dossier América Latina: política y religión
El gobierno de Colombia también reconoció que las relaciones con las principales iglesias y religiones se transferían al derecho público, y esto se reflejó en la posibilidad de firmar dos tipos de contratos, en el campo del derecho público internacional y en el del derecho público nacional.

Por lo tanto, la Corte Constitucional, que examinó el proyecto de asesoría jurídica de la libertad religiosa encontró que el sistema asumía la adopción del régimen especial, diferente del que se utiliza para la libertad pública, asociaciones, comunidades y otras personas jurídicas. Por lo tanto, no es sorprendente la capacidad que tiene el Estado de firmar contratos de derecho público nacional de iglesias y confesiones religiosas, confiriéndoles un estatuto jurídico especial que les permite asistirlas en diversos campos de la vida social, así como en cuestiones de importancia pública, es decir, de Estado, su desarrollo, sobre todo legal y proselitismo, las enseñanzas religiosas, un registro público de las instituciones religiosas, poderes especiales que pertenecen a iglesias, etc.

Las consideraciones confirman lo que fue contenido en el art. I del Concordato. Una novedad es el hecho de que actualmente no tienen aplicación sólo con respecto de la Iglesia católica, sino de todas las religiones en la vida social. No se puede hablar de copiar cualquier norma, lo cual reflejaría el deseo de eliminar cualquier forma de desigualdad - real o aparente - entre las creencias. Considerándolo en una perspectiva más ancha y de forma positiva, las cuestiones que se toman aquí en cuenta conciernen a los casos en los que cada religión que tiene como mínimo estructuras y organizaciones podría introducirse y colaborar con las autoridades públicas. Por lo tanto, la similitud con el Concordato ayudó a allanar el camino para un reconocimiento adecuado y la promoción del derecho a la libertad religiosa. Se confirmó, al mismo tiempo, que en el campo del Concordato las regulaciones son simplemente una expresión de libertad religiosa y pueden, por lo tanto, después de hacer los ajustes necesarios para adaptarse a las características específicas de cada religión, extenderse a todos. Uno de los principales problemas de la sentencia C-027 de 1993 fue reconocerlo contrario a la Constitución, pues daba a la Iglesia católica un monopolio sin la posibilidad de inclusión de otras religiones en estas actividades.

Como resultado, ya no hay normas concordatarias que entonces entraban en conflicto con el principio de igualdad. Tampoco se puede negar que se tiene eficacia legal, ya que estas disposiciones han llegado a ser una parte del derecho aplicado comúnmente en relación con todas las confesiones, también a la Iglesia católica. El punto de apoyo del Concordato es, entonces, no sólo el derecho internacional, sino también el derecho nacional (Ramos Castañeda 2014: 284-291).

Esto tiene una importancia particular en lo que respecta a la cuestión concordataria declarada como inconstitucional después de 1993. La mayoría de ellas todavía son incompatibles con la Constitución, si las aplicamos como normas del Concordato; pues hay que tener en cuenta en muchos casos su importancia como una parte de la legislatura común (Prieto 2010: 32-38). 


\section{Conclusiones}

Colombia tiene una fuerte tradición concordataria. Pese a la situación de los últimos años, el Concordato sigue vigente, e incluso se puede decir que su posición se ha fortalecido entre las ideas controvertidas que aparecieron en relación con la entrada en vigor de la nueva Constitución de 1991. En general, la polémica en torno al Concordato se centró en la adecuación de algunas de sus resoluciones en relación con la ley básica y no en la validez del sistema concordatario como tal. Esto demuestra la aceptación de los supuestos después de 1991 en la Ley de estatuto sobre la libertad religiosa, la legislatura común y la jurisprudencia de la Corte Constitucional.

El sistema concordatario inspiró en Colombia un nuevo modelo de relaciones entre el Estado y las iglesias. En este proceso el Concordato sirvió de fondo para la implantación del principio de cooperación con iglesias.

Después de 1991 muchas de las resoluciones del Concordato se hicieron principios generales o se transfirieron a otras confesiones. Esta evolución es un elemento relevante en el desarrollo de la ley religiosa para todas las religiones. En este sentido legal, el Concordato tiene su lugar, al mismo tiempo sin violar el principio de igualdad, lo cual estaba en la base de su declaración de inconstitucionalidad de 1993.

El sistema colombiano pretende conservar un equilibrio entre las normas del derecho común con un principio de particularidad en relaciones con cada religión. La celebración de acuerdos que tienen mucha importancia en el sistema de actos del derecho nacional también es una garantía de laicidad del país y, a la vez, contribuye a un respeto verdadero y a que se garantiza seguridad legal en el campo de la colaboración del Estado con las religiones, lo cual permite evitar una injerencia excesiva de una de las partes en las actividades tomadas por otra.

Independientemente de lo susodicho, la disputa sobre la constitucionalidad de muchas de las resoluciones del Concordato no se resolvió del todo. Aunque se produjeron modificaciones positivas también a raíz de la evolución de la ley religiosa colombiana, se puede decir que la situación actual sigue siendo insegura desde el punto de vista de la transparencia y seguridad legal.

Diferentes opiniones con respecto a la aplicación de la puesta en marcha de las leyes consideradas inconstitucionales no son fáciles de conciliar. Sin embargo, existe un acuerdo con respecto a que la solución legal tiene que ser precisa y - en la medida de lo posible - estable, basada también en un reinicio de negociaciones entre las partes con el fin de firmar un nuevo tratado o una modificación del presente de acuerdo con los mecanismos del derecho internacional. El nuevo acuerdo estará sujeto a la revisión prevista en el art. 241 de la Constitución. De esta forma, será posible conciliar el acuerdo con las leyes básicas, lo cual permitirá superar inseguridades e incertidumbres que aparecieron en los últimos años. Sin embargo, por ahora no se han tomado medidas ni negociaciones al respecto entre la Santa Sede y el gobierno colombiano.
El Concordato colombiano y la jurisprudencia de la Corte Constitucional

Marta Zuzanna Osuchowska 
Dossier América Latina: política y religión
El punto de partida de esta reforma puede ser el acuerdo firmado en 1992. Sobre la base del mismo vale la pena realizar un análisis exhaustivo del Concordato, teniendo en cuenta el contexto de la nueva ley religiosa de Colombia. Las posibilidades de modificaciones son muchas, por ejemplo, la aprobación de un concordato total o parcial. Independientemente del modo tomado, es preciso un proceso de discernimiento que tome en cuenta lo que ya se proporciona y defina en la ley común y la eliminación o modificación de las resoluciones innecesarias u obsoletas del presente Concordato.

Al mismo tiempo se pueden plantear algunas cuestiones sin arreglar hasta la fecha que han cobrado importancia en los últimos años como, por ejemplo, la objeción de conciencia, el respeto por la enseñanza y una educación de acuerdo con sus propias convicciones, temas bioéticos, símbolos religiosos en lugares públicos, nuevas regulaciones concernientes al financiamiento de la Iglesia católica, etc. El Concordato puede llegar a ser no sólo un instrumento de colaboración con una religión y garantizar derechos exclusivamente para sus fieles, sino que también debe ser una puerta que asegure acceso a un mayor desarrollo de la ley de libertad religiosa.

\section{Bibliografía}

Constitución política de Colombia. 1991, “Gaceta Constitucional”, no 116 de 20 de julio de 1991.

Convención de Viena sobre Derecho de los Tratados. Convención de Viena sobre el derecho de los tratados. U.N. Doc A/CONF.39/27 (1969), 1155 U.N.T.S. 331.

Ley 20 de 1974, "Diario oficial", no 34.234, de 14 de enero de 1975 por el cual se prueba el "Concordato y el Protocolo Final entre la República de Colombia y la Santa Sede" suscrito en Bogotá el 12 de julio de 1973.

Eguren J. A. (1974), Derecho Concordatario Colombiano, Librería Stella, Bogotá.

González F. E. (2002), La iglesia en el siglo XX. Las reformas al Concordato, "Credencial Historia", no 153, disponible en: http://www.banrepcultural.org/blaavirtual/revistas/credencial/ septiembre2002/laiglesiaXX.htm, fecha de consulta: 30.04.2016.

Holguín A. (1973), Análisis del Nuevo Concordato, ed. de la Rev. Derecho Colombiano, Bogotá.

Lozano Simonelli F. (1981), El Concordato Colombiano de 1973, Banco de la República, Bogotá.

Martín de Agar J. T., Uribe Blanco M., (1998), Concordato y jurisprudencia constitucional en Colombia, en: La libertad religiosa y de conciencia ante la justicia constitucional, Actas del VIII Congreso Internacional de Derecho Eclesiástico del Estado, Granada 13-16 maja 1997.

Martín de Agar J. T. (2000), Raccolta di Concordati (1950-1999), Libreria Editrice Vaticana, Città del Vaticano, Vaticano.

Maya Barroso D. E. (2008), La laicidad del Estado colombiano, „Criterios - Cuadernos de Ciencias Jurídicas y Política Internacional", vol. 1, no 2. 
Monroy Cabra M. G. (1975), Régimen Concordatario Colombiano, Temis, Bogotá.

Monroy Cabra M. G. (2001), Análisis de la jurisprudencia constitucional en materia de Tratados Públicos en la Constitución de 1886 y en la de 1991, en: La Constitución por conseguir: balance de una década de cambio institucional, Bogotá.

Navarro Floria J. (2011), Acuerdos y concordatos entre la Santa Sede y los países americanos, Editorial de la Universidad Católica Argentina, Buenos Aires.

Preciado Agudelo D. (1989), El Concordato, Librería del Profesional, Bogotá.

Prieto V. (1996), Iglesia Católica y libertad religiosa en Colombia. La Libertad Religiosa, en: Memoria Del IX Congreso Internacional De Derecho Canónico, México.

Prieto V. (1998), El Concordato en la jurisprudencia colombiana, Universidad de La Sabana, Bogotá.

Prieto V. (2001), El Concordato colombiano de 1973, en: Actas del Congreso Latinoamericano sobre libertad religiosa, Lima.

Prieto V. (2008), Libertad religiosa y confesiones (Derecho Eclesiástico del Estado Colombiano), Universidad de la Sabana, Bogotá.

Prieto V. (2010), El Concordato de 1973 y la evolución del derecho eclesiástico colombiano. Situación actual y perspectivas de futuro, "Revista General de Derecho Canónico y Derecho Eclesiástico del Estado", no 22.

Ramos Castañeda F.A. (2014), Derecho fundamental de libertad religiosa constitucional y jurisprudencial de la República de Colombia: análisis histórico y régimen jurídico, Universidad San Dámaso, Madrid.

Restrepo Uribe L. (1973), El concordato, ayer - hoy: 1887 - 1973, Ediciones Paulinas, Bogotá.

Vázquez Carrizosa A. (1973), El concordato de Colombia con la Santa Sede: Julio 12 de 1973, Impreso en Italgraf, Bogotá.

Vázquez Carrizosa A. (1993), El Concordato de 1973 de Colombia con la Santa Sede ante el Derecho internacional y la Constitución de 1991, „Revista de la Academia Colombiana de Jurisprudencia", no 300-301.
El Concordato colombiano y la jurisprudencia de la Corte Constitucional

Marta Zuzanna Osuchowska 